\title{
Spatial navigation impairment in mice lacking cerebellar LTD: a motor adaptation deficit?
}

Eric Burguière(1), Angelo Arleo(1) \&, Mohammad R. Hojjati(2)(3), Ype Elgersma(2), Chris I. De Zeeuw(2), Alain Berthoz(1) and Laure Rondi-Reig(1)

(1) Laboratoire de Physiologie de la Perception et de l'Action, UMR CNRS 7152, 11 place Marcelin Berthelot, Collège de France, 75005 Paris, France.

(2) Department of Neuroscience, Erasmus MC, 3000 DR, Rotterdam, The Netherlands.

(3) Department of Physiology, Shahrekord University of Medical Sciences, IRAN

${ }^{\&}$ Current address: Neuroscience Group, Sony Computer Science Laboratory, 6 rue Amyot, 75005 Paris, France.

Correspondence should be addressed to LRR (laure.rondi-reig@college-de-france.fr) 


\begin{abstract}
L7-PKCl transgenic mice, having a specific lack of parallel fiber-Purkinje cell LTD, were tested with two different mazes to dissociate the relative importance of declarative and procedural components of spatial navigation. Our data bring evidence for a deficit of L7-PKCI mice in the acquisition of an adapted goal-oriented behavior, i.e. in the procedural component of the task. This finding supports the hypothesis that cerebellar LTD may subserve a general sensory-motor adaptation process shared by motor and spatial learning functions.
\end{abstract}


Spatial navigation offers a suitable framework to study the ability of animals to adapt their behavior to a specific context, defined here as the combination of the multimodal information sensed by the animal and its internal state at a specific time. Spatial navigation requires at least two complementary processes: (i) the elaboration of a spatial representation of the environment (declarative component), enabling the animal to encode the spatio-temporal relationships among environmental cues or events; (ii) the acquisition of a motor behavior adapted to the context in which navigation takes place (procedural component), permitting the execution of optimal (direct) trajectories toward rewarding locations ${ }^{1}$. Several types of cerebellar animal models have been tested in spatial navigation tasks ${ }^{2}$ (Supplementary Note). The consensus that emerged from these studies points toward a role of the cerebellum in mediating the procedural component of the spatial navigation function. We focused on the cellular mechanisms subserving the contribution of the cerebellum in spatial learning. Our working hypothesis is that cerebellar Long-Term synaptic Depression (LTD), occurring at the parallel fiber-Purkinje cell (PF-PC) synapses and required for the acquisition of classical conditioning tasks ${ }^{3}$, may also be necessary for the acquisition of efficient trajectories toward a spatial goal through a basic and common process of sensory-motor adaptation.

We employed the L7-PKCl transgenic mice model ${ }^{4}$, which presents a specific inactivation of the PF-PC LTD, to investigate the potential role of this cellular mechanism during spatial navigation (see Supplementary Methods; all experiments were performed in compliance with the European ethical animal committee). L7-PKCI mutants are known to have intact motor capabilities and normal electrophysiological properties of Purkinje cells ${ }^{5,6}$ (see Supplementary Note). Likewise, we observed no abnormalities in the sensory-motor reflexes, physical characteristics, and general behavior of L7-PKCl mice (Supplementary Table 1). Hippocampal functions (synaptic transmission and plasticity), known to be essential for navigation tasks, appeared also to be conserved in L7-PKCI mice (Supplementary Fig. 1).

In order to dissociate the relative importance of the declarative and procedural components of navigation, we adopted two different behavioral paradigms: the Morris water maze (MWM) and a new task called the Starmaze (Fig. 1). In both cases, the animal has to find a fixed hidden platform from random departure locations, which requires the declarative capability of learning a spatial representation of the environment. Yet, in contrast to the MWM task, the Starmaze allows the animal to 
only swim within alleys guiding its movements. This helps to execute goal-directed trajectories effectively, and reduces the procedural demand of the task.

To compare the navigation performances of L7-PKCI mice $(n=14)$ and their control littermates $(n=15)$ when solving the hidden-platform version of the MWM, we first used three standard parameters: (i) The mean escape latency, measuring the time employed by the animal to reach the target and estimates its ability to learn the navigation task. (ii) The search score, describing the goal-oriented trajectory quantitatively $^{7}$. (iii) The circling behavior, defined as the time spent in a $10 \mathrm{~cm}$ annulus near the wall of the pool, already interpreted as a deficit in the procedural component of the spatial task ${ }^{8}$.

Both, the mean escape latency and the search score of L7-PKCl mice were significantly higher than those in wild types (ANOVA, $F_{1,27}=14.2, P<0.001$; and ANOVA, $F_{1,27}=19.3, P<0.001$ ) (Fig. 2a). The escape latency measure and the search score method were highly correlated for both groups of animals $(R=0.966, P$ $<0.001$, and $R=0.942, P<0.001$, for wild types and mutants respectively) (Fig. 2b). The circling times of the LTD-deficient mutants were also significantly longer than those of wild types (ANOVA, $F_{1,27}=11.6, P<0.01$ ) (Fig. 2c). These results showed that $\mathrm{L} 7-\mathrm{PKCl}$ mice were impaired in solving the MWM task. This spatial navigation impairment was due to neither a deficit in swimming speed (ANOVA, $F_{1,27}=0.46, P>$ 0.5 ) (Fig. 2d) nor a deficit in visual guidance abilities (ANOVA, $F_{1,27}=1.2, P>0.1$ ) (Fig. 2e).

The ratio between the time spent in the target quadrant and the duration of the trial provides an estimate of the ability of an animal to locate the platform. Both groups of mice spent significantly longer time in the target quadrant than in any other quadrant (ANOVA, $F_{1,9}=15, P<0.0001$ ), which suggested that both control and L7$\mathrm{PKCl}$ mice were able to acquire a memory of the localization of the platform. However, an inter-group comparison of this ratio as a function of learning showed that L7-PKCl mice spent significantly less time than controls within the target quadrant (ANOVA, $\left.F_{1,27}=5.1, P<0.05\right)$ (Fig. 2f). To investigate this difference and to assess the accuracy of the mice goal-oriented behavior during learning, we calculated the average mouse-to-platform distance during a trial for both control and L7-PKCl mice (Fig. 2g). L7-PKCI mice presented a longer mean distance relative to the platform than control animals over the entire training period (ANOVA, $F_{1,27}=16.6$, $P<0.001)$. This result indicated that the trajectories of $\mathrm{L} 7-\mathrm{PKCl}$ mice toward the platform were less direct compared to those employed by control mice. This issue 
was further investigated by computing the ongoing egocentric angle $\left(\phi(t) \in\left[0^{\circ}, 180^{\circ}\right]\right)$ between the optimal direction towards the target and the actual motion direction of the animal. The larger the angle $\phi$, the bigger the deviation between the ideal goaldirected trajectory and the actual trajectory. Both groups significantly decreased their mean angular deviation from the ideal trajectory over training (ANOVA, $F_{1,9}=29.4, P$ $<0.0001$, see Fig. 2h). However, L7-PKCl mice exhibited significantly higher deviations than control mice (ANOVA, $F_{1,27}=14.9, P<0.001$ ). The statistical correlation between the mean angular deviation measure and the search score parameter was significantly high for both wild types and mutants $(R=0.871, P<$ 0.001 , and $R=0.839, P<0.001$, respectively).

The results obtained with the MWM may suggest that L7-PKCl mice could learn to locate the platform (declarative component) but they executed non-optimal goal-directed trajectories (procedural component) (see Supplementary Fig. 2 for a qualitative comparison between the searching behavior of controls and mutants over training). To test this hypothesis we employed a task requiring declarative capabilities but with a lower procedural demand than the MWM: the allocentric Starmaze task (see Supplementary Methods). This task, similar to the MWM, has been shown to depend on an intact hippocampal function (L. Rondi-Reig et al., Soc. Neurosci. Abstr., 329.2, 2004). To assess the ability of mice to learn the Starmaze task, the number of visited alleys and the mean distance swam before finding the platform were observed. Both L7-PKCl mice $(n=15)$ and control mice $(n=11)$ improved significantly their ability to quickly reach the platform (ANOVA, $F_{1,24}=7.45, P<$ 0.0001). No statistical difference was observed between the two groups concerning the number of visited alleys (ANOVA, $F_{1,24}=0.64, P>0.1$, Fig. 3a) and the mean distance swam to reach the platform (ANOVA, $F_{1,24}=2.56, P>0.1$, Fig. $3 b$ ) (see Supplementary Fig. 3 for a qualitative representation of the similar behavior of controls and mutants).

The results obtained with the Starmaze strengthened our hypothesis that the declarative component was not affected in L7-PKCI mice. The absence of a deficit when the trajectory was guided corroborated the results obtained with the MWM, which pointed towards the inability of L7-PKCl mice to adapt their goal-oriented behavior effectively.

The parallel fiber-Purkinje cell (PF-PC) LTD mechanism is likely to constitute a core process underlying cerebellar learning, and it has been proposed to contribute 
to both motor and cognitive learning ${ }^{9}$. It has been demonstrated that $27-\mathrm{PKCl}$ mutants have response deficits in both adaptation of the vestibulo-ocular reflex ${ }^{4}$ and eyelid conditioning tasks ${ }^{10}$. In addition, cerebellar LTD seems most prominently involved in rapid learning of well-timed movements with specific amplitudes. Our results corroborated previous spatial navigation studies with other cerebellar models ${ }^{8,11}$, and suggested a participation of the PF-PC LTD in the procedural component of navigation (Supplementary Note). How could the same cellular mechanism, i.e. PF-PC LTD, be involved in motor learning and more cognitive process such as spatial learning? Several cognitive processes can be considered on the basis of the same sensory-motor coupling scheme observed in classical motor learning ${ }^{12}$. Spatial navigation requires a linkage between the spatial context (including sensory inputs and internal state information) and the explorative response (motor output) characterized by the animal's trajectory. While the spatial context may be conveyed by the mossy fiber - granule cell - parallel fiber pathway relaying information from the pontine nuclei ${ }^{13}$, errors in the explorative response may be mediated by the climbing fiber signals that originate from the olivary subnuclei that are innervated by descending projections from the mesodiencephalic junction and cerebral cortex ${ }^{14}$. Since induction of PF-PC LTD requires conjunctive activation of the parallel fiber and climbing fiber pathways, this form of synaptic plasticity could be responsible for the establishment of this linkage ${ }^{12}$. Thus the cerebellum may mediate a general learning function to create a context-response linkage adapted to the task $^{15}$. During spatial learning, the subject could establish an appropriate contextresponse coupling resulting in effective motor behavior (e.g., in the execution of optimal trajectories to the target). At the cerebellar level, procedural learning may result from a classical control learning scheme in which the feedback loop allows the system to converge towards an adapted context-motor linkage (Supplementary Fig. 4). The absence of PF-PC LTD in L7-PKCI mice could result from the accumulation of errors over time (i.e., during the execution of a goal-directed trajectory), due to the absence of continuous context-dependent corrections of the motor signals. From a behavioral point of view, the accumulation of these errors would lead to a drift during unconstraint (not guided) navigation as in the MWM task, whereas it would be irrelevant in the Starmaze task due to the reduced number of possible goal-directed trajectories. 


\section{Acknowledgments}

The authors thank M. Rutteman, V. Gautheron and F. Maloumian for technical supports. L.R.-R. is supported by the $\mathrm{ACl}$ 'Neurosciences Intégratives et Computationnelles' (NIC 0083), the IFR 52 'Transduction du signal: Molécules à Action centrale et périphérique', and the 'GIS Longévité' (grant L0201). A.B. is supported by the European Laboratory of Neurosciences and Action (LENA). C.I.D.Z. is supported by NWO, EEC, and Neuro-Bsik. 


\section{References}

1. Schenk, F. \& Morris, R. G. Exp. Brain Res. 58, 11-28 (1985).

2. Rondi-Reig, L. \& Burguiere, E. Prog. Brain Research 148, $201-212$ (2004).

3. Thompson, R. F. et al. Int. Rev. Neurobiol. 41, 151-189 (1997).

4. De Zeeuw, C. I. et al. Neuron 20, 495-508 (1998).

5. Goossens, H. H. et al. Eur. J. Neurosci. 19, 687-697 (2004).

6. Goossens, J. et al. J. Neurosci. 21, 5813-5823 (2001).

7. Petrosini, L., Molinari, M., \& Dell'Anna, M. E. Eur. J. Neurosci. 8, 1882-1896 (1996).

8. Leggio, M. G. et al. Exp. Brain Res. 127, 1-11 (1999).

9. Ito, M. Physiol Rev. 81, 1143-1195 (2001).

10. Koekkoek, S. K. et al. Science 301, 1736-1739 (2003).

11. Martin, L. A., Goldowitz, D., \& Mittleman, G. Eur. J. Neurosci. 18, 2002-2010 (2003).

12. Ito, M. Rev. Neurol. (Paris) 149, 596-599 (1993).

13. Thach, W. T., Goodkin, H. P., \& Keating, J. G. Annu. Rev. Neurosci. 15, 403442 (1992).

14. De Zeeuw, C. I. et al. Trends Neurosci. 21, 391-400 (1998).

15. Thach, W. T. Int. Rev. Neurobiol. 41, 599-611 (1997). 


\section{Figure 1}

Comparison of two paradigms of spatial navigation. The Morris water maze (MWM) (a) and the allocentric version of the Starmaze task (b). Both navigation tasks require to find an escape platform (dashed circle) submerged under opaque water. To locate the platform efficiently, animals have to employ the configuration of cues located outside the apparatus. In both paradigms animals are trained to reach the platform from four randomly selected departure points (black stars). Similar to the MWM, solving the Starmaze task implies spatial learning capabilities. In contrast, animals are constraint to swim within alleys that guide their movements, which reduces the possible deviations from an ideal trajectory.

\section{Figure 2}

Inactivation of LTD in L7-PKCl mice affected their performance in the MWM task. (a) The mean escape latencies of controls and mutants (white and black squares, respectively) were similar at the beginning of training (day 1). However, controls improved their performance significantly better than mutants over time. The behavioral patterns corresponding to the search scores are illustrated by the cartoon trajectories on the right side. The lower the score, the better the searching behavior. The search scores of $\mathrm{L} 7-\mathrm{PKCl}$ mice were significantly higher than those of control animals. (b) The search scores and the escape latencies were highly correlated for both control (top) and mutant (bottom) mice, suggesting that the longer time-to-goal needed by L7-PKCI mice was due to non-optimal searching trajectories. (c) L7-PKCl mutants exhibited a significantly larger amount of circling behavior over training, previously interpreted as a deficit in the procedural component of navigation ${ }^{7}$. (d) The mean swimming speeds of controls and mutants remained comparable over the entire training period. (e) Mutants are not impaired when solving the visible platform version of the MWM (i.e., visuo-guidance navigation task). (f) The mean ratio between the time spent within the target quadrant and the total duration of the trial of both controls and mutants increased significantly above the random trajectory level during training. However, controls improved their ratio significantly better than L7$\mathrm{PKCl}$. (g) The mouse-platform distance parameter demonstrated that mutants followed significantly longer trajectories than controls. (h) The mean angular 
deviation between ideal and actual trajectory showed that mutants had a deficit in maintaining their body locomotion oriented toward the platform during navigation.

\section{Figure 3}

L7-PKCl mutants were not impaired in solving the allocentric version of the Starmaze task. (a) The mean number of alleys visited during a trial was not significantly different between control and mutant mice. (b) The distance swam to reach the target was not significantly different between the two groups. 

a

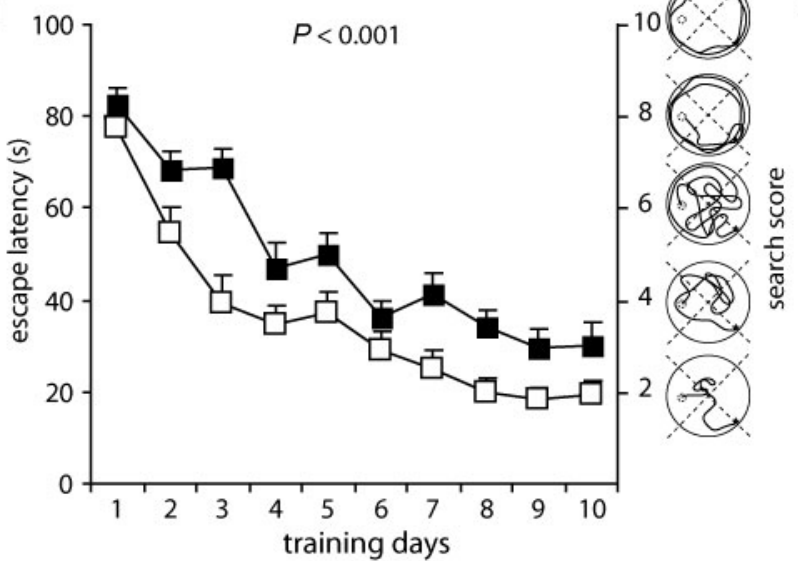

ロ--/- - - -/L7-PKCI

C

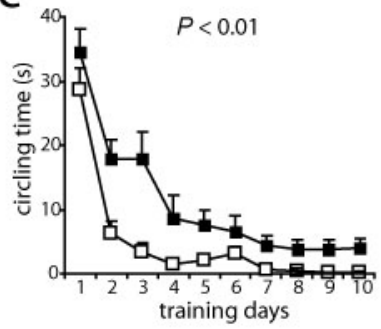

f

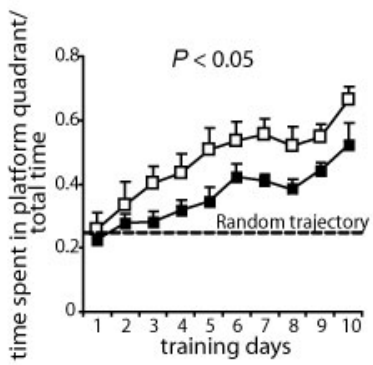

d

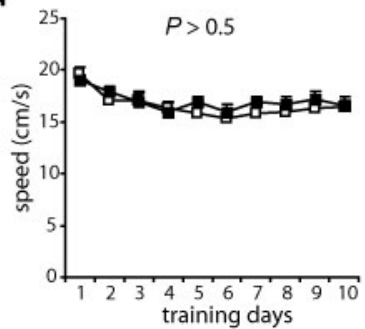

g

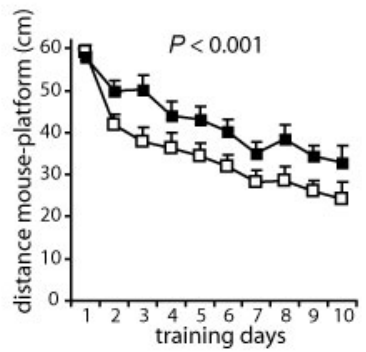

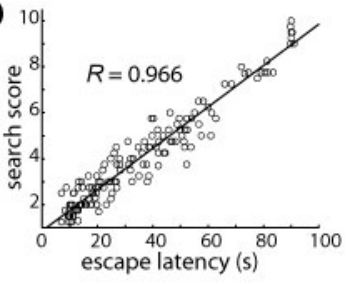

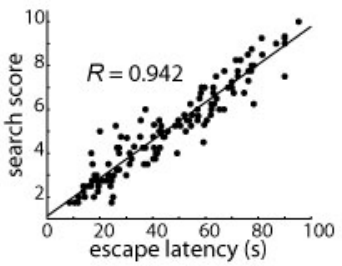

e

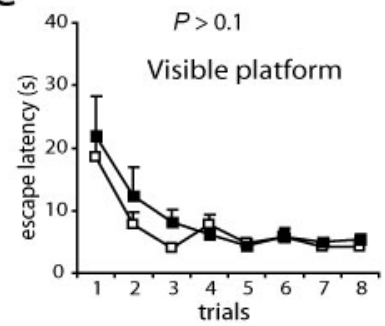

h

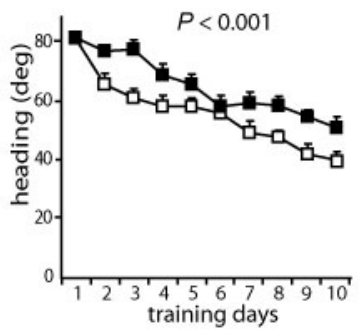



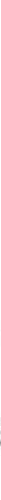


\section{SUPPLEMENTARY METHODS}

\section{Experimental subjects}

Heterozygous transgenic L7-PKCI (-/L7-PKCl) mice and their wild-type (-/-) littermates were used for this study. In the L7-PKCI mouse, the pseudosubstrate PKC inhibitor, $\mathrm{PKCl}$, is selectively expressed in Purkinje cells under the control of the pcp2 (L7) gene promoter ${ }^{1}$. All animals were bred in a C57BL/6 mouse strain background and were housed in standard conditions (12h light/12h dark, water and food ad libitum). Both control and transgenic L7-PKCI mice underwent experiments between 3.5 to 6 months. All the behavioral analyses were performed blind with respect to the genotype of the mice. All experiments were performed in compliance with the European ethical animal committee.

\section{Behavioral experiments}

According to the S.H.I.R.P.A. protocol for comprehensive phenotype assessment ${ }^{2}$, all animals were tested by a series of experiments run sequentially. The first screen was a set of behavioral tests concerning general appearance and neurological reflexes. The second screen aimed to detect eventual motor, equilibrium or anxiety differences between control and mutant mice. The third screen assessed their spatial navigation abilities. We tested 14 mutants and 15 controls for the Morris water maze task, and 15 mutants and 11 controls underwent the starmaze task.

\section{General appearance and neurological reflexes}

Animals were tested for gross abnormalities that could interfere with their behavior in the navigation tasks. General appearance was evaluated by observing the presence of whiskers, the absence of palpebral closure and pilo-erection. Body weight was recorded. Each mouse was then placed for three minutes in an unfamiliar standard mouse cage for observation of any abnormal spontaneous behavior. Examples of aberrant actions include wild running, abnormal jumping or grooming, and frozen immobility. Three neurological reflexes were studied according to established protocols ${ }^{3}$. Approaching the eye and touching the ear with the tip of a clean cotton swab tested the eyeblink reflex and the ear twitch reflex, respectively. The whisker-orienting reflex was tested by lightly brushing the whiskers of a freely moving animal with a small paintbrush. 


\section{Basic motor skills}

A series of tests was carried out in order to compare control and L7-PKCl transgenic mice in terms of motor and equilibrium abilities.

Muscle strength was tested by hanging the mouse by its two anterior paws on a rod (25 cm long, $0.3 \mathrm{~cm}$ in diameter) located $1 \mathrm{~m}$ above a thick carpet to cushion falls. The time the animal hung on a wire without falling was recorded ${ }^{4}$.

To quantify dynamic balance, we used the rotarod apparatus in a fixed position. The rotarod apparatus consists of a wooden horizontal rod $3 \mathrm{~cm}$ in diameter and $50 \mathrm{~cm}$ long. The animal was placed at the middle of the non rotating rod, its body axis perpendicular to the rod longitudinal axis. The time the animal remained on the rod was recorded. Each trial ended either when the animal fell or after $180 \mathrm{~s}$ (in accordance with the rotarod protocol). This test measures the dynamic balance because the animal can anticipate and even prevent any fall ${ }^{4}$. We used an unsteady platform ${ }^{5}$ to quantify static balance. Mice had to remain still on a narrow unsteady elevated platform, which could tilt by $30^{\circ}$ in every direction. The slip frequency on the board was calculated for three trials per animal.

To assess both exploratory behavior and motor coordination, a hole-board was designed ${ }^{4}$ by deriving it from the one used by ${ }^{6}$ with cerebellar mutant mice. It consists of an experimental box (35 × $35 \times 25 \mathrm{~cm})$, with a raised $(2 \mathrm{~cm})$ platform in which 36 holes ( $2 \mathrm{~cm}$ in diameter, arranged in a $6 \times 6$ array) have been drilled. The mouse was placed in the middle of the platform and its behavior was video-recorded. Walking time and number of stumbles were counted. To control the possibility that mice stumbled more often simply because they were more active on the hole-board, the number of stumbles per minute of walking time was calculated. This stumble frequency was considered as a measure of motor coordination?

\section{Anxiety test}

The classical elevated "+" maze ${ }^{8}$ was employed for this test. It is a crossshaped maze with two high-walled arms and two wall-less arms. Each arm is $24 \mathrm{~cm}$ long and $8 \mathrm{~cm}$ wide. Walls are $20 \mathrm{~cm}$ high. The whole apparatus was elevated $1 \mathrm{~m}$ above the floor. Previous studies have shown that anxious mice spend more time in the walled arms rather than in the open ones. Anxiolytic treatment significantly increases the number of entries in open arms, whereas anxiogen reduces $i t^{9}$. We measured the percentage of number of entries and the percentage of time spent by 
the animals in the open arms. An entry was considered effective when the animal placed its four paws in the arm. The test lasted 5 min.

\section{Navigation tasks}

Two different apparatus were employed for the navigation tasks: the Morris water maze ${ }^{10}$ and the allocentric version of the Starmaze task (L. Rondi-Reig et al., Soc. Neurosci. Abstr., 329.2, 2004) (Fig. 1).

The Morris water maze task.

Mice were trained in a circular water tank (150 cm in diameter, $40 \mathrm{~cm}$ high) to find an escape platform (10 cm of diameter) hidden $1 \mathrm{~cm}$ below the surface of the water at a fixed location (Fig. 1a, NW quadrant). The pool contained water $\left(21^{\circ} \mathrm{C}\right)$ made opaque by the addition of an inert and nontoxic product (Accusan OP 301). Both the pool and the surrounding distal cues were kept fixed during all experiments. Each mouse underwent one training session per day consisting of four trials. The starting position (North, East, West, or South) was randomly selected with each quadrant sampled once a day. At the beginning of each trial, the mouse was released at the starting point (black stars in Fig. 1a) and made facing the inner wall. Then, it was given a maximum of $90 \mathrm{~s}$ to locate and climb onto the escape platform. If the mouse was unable to find the platform within the $90 \mathrm{~s}$ period, it was taken to the platform by the experimenter. In either case, the mouse was allowed to remain on the platform for $30 \mathrm{~s}$. Animals received 40 training trials over 10 days.

The measured parameters were: (i) the average time necessary to reach the platform (i.e., mean escape latency); (ii) the search score ${ }^{11}$; (iii) the average speed; (iv) the amount of circling (i.e., the time spent in a $10 \mathrm{~cm}$ annulus near the wall of the pool); ( $v$ ) the mean distance of the mouse relative to the platform, averaged over trials as well as over the entire training period; (vi) the ratio between the time spent in the target quadrant (i.e., the quadrant containing the platform) and the duration of the trial; (vii) the "mean angular deviation" (i.e., the angle between the optimal direction towards the target and the actual motion direction of the animal). For each trial, we computed the mean deviation $\bar{\phi}_{i}=\langle\phi(t)\rangle_{t \in\left[0, T_{i}\right]}$, where $i$ is the trial and $T_{i}$ denotes the duration of the trial. Then, we averaged over all the trials performed in one day by all the subjects of the same group of animals. 
After training, mice underwent the visible platform task. A proximal cue was placed on the top of the submerged platform, which was positioned at one of four possible locations (North, East, West, and South). To solve this task, animals had to learn that the proximal cue (beacon) indicated the location of the platform. The starting position of the animal was fixed and located at the center of the circular water tank. The test was performed during two consecutive days with four trials a day. The four possible locations of the platform were randomly sampled each day. The time needed by the mice to reach the platform was measured.

The allocentric (multiple departures) version of the Starmaze task.

The Starmaze is an aquatic maze with five alleys forming a central pentagonal ring with a side distance of $37 \mathrm{~cm}$ and five peripheral alleys radiating from this pentagonal ring (Fig. 1b). Each radial alley is $47 \mathrm{~cm}$ long and $25 \mathrm{~cm}$ wide. The entire maze (204 cm diameter) is filled with water that has been made opaque with an inert and nontoxic product (Accuscan OP 301). The maze was placed at a fixed location inside a large room $\left(50 \mathrm{~m}^{2}\right)$ with fixed $3 \mathrm{D}$ extra-maze cues. We used a randomdeparture protocol (similar to that employed for the Morris water maze) lasting 13 days and forcing the animal to use an allocentric strategy to perform the task. The platform was always located at the end of alley 7 . The mouse was placed at the end of a randomly selected radial arm not containing the platform (i.e. 1, 3, 5, and 9). Each mouse was given four trials per day, each trial corresponding to a different departure arm. If an animal was not able to find the platform within a $90 \mathrm{~s}$ period, it was taken to the platform by the experimenter. In either case, the animal was left for $30 \mathrm{~s}$ on the platform. The measured parameters were the distance traveled and the number of alleys visited by the animal in order to find the platform.

\section{Electrophysiological recording}

Sagittal hippocampal slices $(400 \mu \mathrm{m})$ were placed in a submerged 4-chamber recording set-up and perfused continuously at a rate of $1.5 \mathrm{~mL} / \mathrm{min}$ with artificial cerebrospinal fluid (ACSF) equilibrated with $95 \% \mathrm{O}_{2}, 5 \% \mathrm{CO}_{2}$ at $30^{\circ} \mathrm{C}$. ACSF contained (in mM) $120 \mathrm{NaCl}, 3.5 \mathrm{KCl}, 2.5 \mathrm{CaCl}_{2}, 1.3 \mathrm{MgSO}_{4}, 1.25 \mathrm{NaH}_{2} \mathrm{PO}_{4}, 26$ $\mathrm{NaHCO}_{3}, 10 \mathrm{D}$-glucose. Extracellular recordings of field excitatory postsynaptic potentials (fEPSPS) were made in CA1 stratum radiatum with Pt/lr electrodes (FHC, Bowdoinham, ME). In all experiments, a bipolar Pt//r electrode was used to stimulate the Schaffer collateral/commissural afferents prodromically every minute. All stimulus 
pulses were $100 \mu \mathrm{s}$ in duration and one- $(100 \mathrm{~Hz})$ or two-thirds (theta burst LTP) of the maximum fEPSP, which was assessed first by an input-output curve. Average stimulation strength and baseline fEPSP were similar between genotypes for all of the experiments conducted. For LTP, statistical comparisons were made using the number of slices as sample size.

\section{Data acquisition and statistical analysis}

Data acquisition was performed by means of a video recording system and a tracking software. Data processing (e.g., the computation of the assessed parameters) was automated via a MATLAB batch program developed in our laboratory. Data were analyzed with the Statview 5.0 software by means of $T$-test and repeated measure analysis of variance (ANOVA). The significant threshold of tests was fixed at $5 \%$ ( $p<0.05$ was significant). All data are presented as mean \pm S.E.M.

\section{References}

1. De Zeeuw, C. I. et al. Neuron 20, 495-508 (1998).

2. Crawley, J. N. Neuropeptides 33, 369-375 (1999).

3. Paylor, R. et al. Learn. Mem. 5, 302-316 (1998).

4. Rondi-Reig, L. et al. Neuroscience 77, 955-963 (1997).

5. Hilber, P., Lalonde, R., \& Caston, J. J. Neurosci. Methods 88, 201-205 (1999).

6. Guastavino, J. M. Physiol Behav. 32, 225-228 (1984).

7. Rondi-Reig, L. \& Mariani, J. Brain Res. Bull. 57, 85-91 (2002).

8. Pellow, S. et al. J. Neurosci. Methods 14, 149-167 (1985).

9. Lister, R. G. Psychopharmacology (Berl) 92, 180-185 (1987).

10. Morris, R. et al. Nature 297, 681-683 (1984).

11. Petrosini, L., Molinari, M., \& Dell'Anna, M. E. Eur. J. Neurosci. 8, 1882-1896 (1996). 


\section{SUPPLEMENTARY NOTES}

\section{Previous studies with cerebellar models.}

Several types of cerebellar animal models have been tested in spatial navigation tasks: mutant mice ${ }^{1}$, hemicerebellectomized rats $^{2}$, and rats with cerebellar input lesions $^{3}$. All cerebellar animals showed impaired learning capabilities when tested with the classical Morris water maze, a task in which the animal has to find a fixed hidden platform from either multiple or a unique starting position ${ }^{4}$. The consensus that emerged from these studies points toward a role of the cerebellum in mediating the procedural component of the spatial navigation function ${ }^{5}$. Likewise, recent data suggested that the procedural impairment observed in cerebellar animals could also induce a more cognitive impairment since the acquisition of a declarative spatial memory relies on the learning of a correct explorative behavior ${ }^{6-8}$.

\section{Electrophysiological properties of the L7-PKCI mouse model.}

De Zeeuw and colleagues have demonstrated that adult L7-PKCI mutant mice have intact baseline discharges of Purkinje cells and no persistent multiple climbing fiber innervation ${ }^{9}$. In fact, even during an optokinetic reflex task, which can be used during visuo-vestibular training, both simple spike and complex spike responses of Purkinje cells appear normal ${ }^{10}$.

\section{Procedural learning and PF-PC LTD mechanism.}

Procedural learning can be defined as the process that permits to execute a new action properly ${ }^{11}$. In particular, this action has to be optimal with respect to the task to be solved. Our hypothesis of a role of PF-PC LTD in the procedural component of navigation was inspired by the theory that considers the cerebellar learning process as an error based system ${ }^{12-14}$. Marr-Albus-Ito theory postulates that the climbing fibers convey error information to the parallel fiber - Purkinje cell synapses and trigger a modulation of their strength via the LTD mechanism. Ito showed that in VOR adaptation experiments PF-PC LTD is likely to constitute the neural substrate of such an error-driven motor learning process ${ }^{15}$. In the case of spatial navigation, the trajectory performed by the animal must be adapted to the spatial context and optimized to lead directly to the goal. 


\section{References}

1. Lalonde, R. \& Botez, M. I. Brain Res. 398, 175-177 (1986).

2. Petrosini, L., Molinari, M., \& Dell'Anna, M. E. Eur. J. Neurosci. 8, 1882-1896 (1996).

3. Rondi-Reig, L. et al. Behav. Brain Res. 132, 11-18 (2002).

4. Petrosini, L., Leggio, M. G., \& Molinari, M. Prog. Neurobiol. 56, 191-210 (1998).

5. Leggio, M. G. et al. Exp. Brain Res. 127, 1-11 (1999).

6. Gandhi, C. C. et al. Behav. Brain Res. 109, 37-47 (2000).

7. Joyal, C. C., Strazielle, C., \& Lalonde, R. Behav. Brain Res. 122, 131-137 (2001).

8. Mandolesi, L. et al. Eur. J. Neurosci. 18, 2618-2626 (2003).

9. Goossens, J. et al. J. Neurosci. 21, 5813-5823 (2001).

10. Goossens, H. H. et al. Eur. J. Neurosci. 19, 687-697 (2004).

11. Hikosaka, O. et al. Trends Neurosci. 22, 464-471 (1999).

12. Marr, D. J. Physiol 202, 437-470 (1969).

13. Albus, J. Math. Biosci. 10, 26-61 (1971).

14. Ito, M. (1984).

15. Ito, M. \& Kano, M. Neurosci. Lett. 33, 253-258 (1982). 


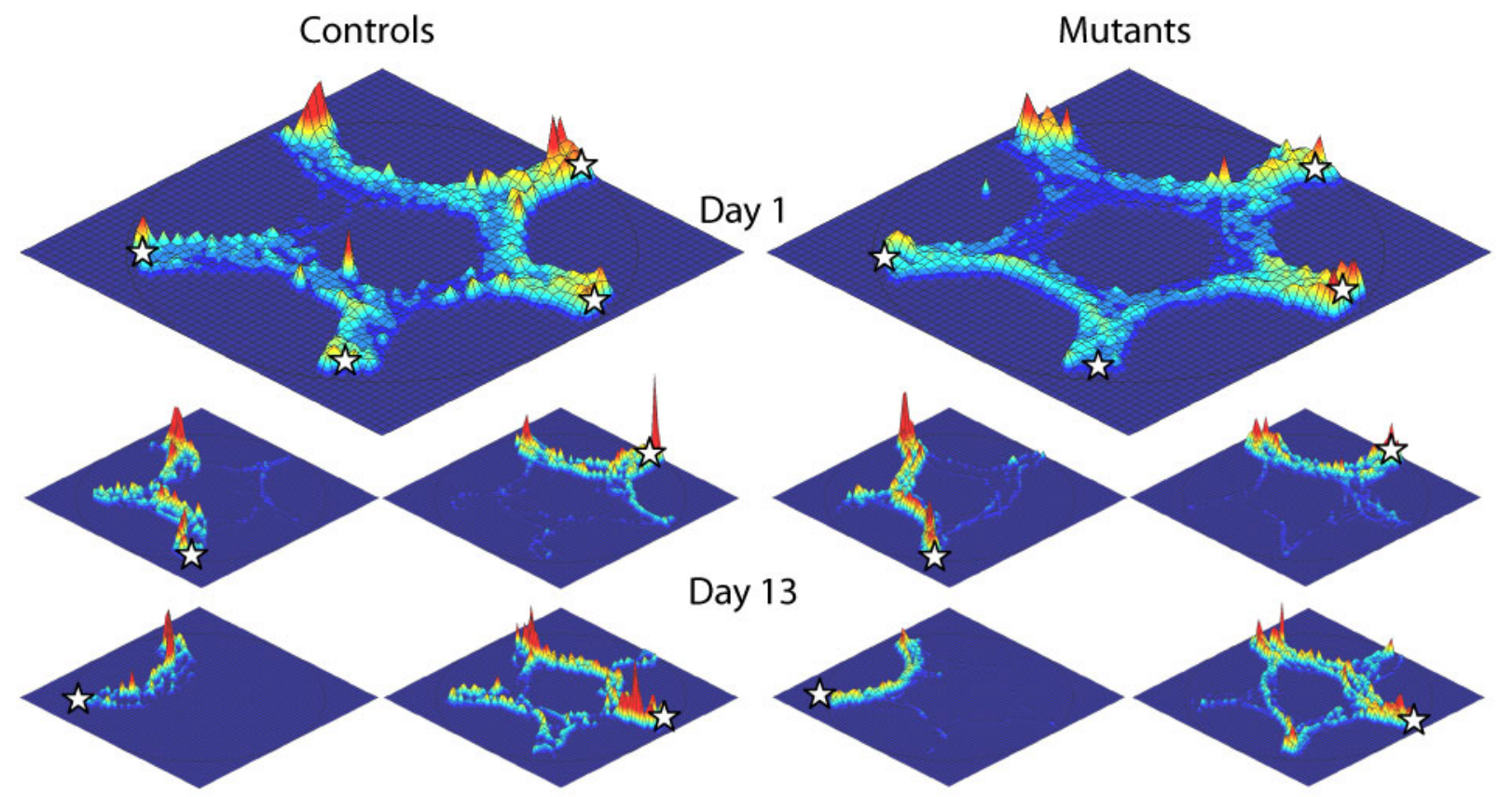

Three-dimensional diagrams showing the mean time spent by the two groups of animals at each location of the Starmaze at the beginning (day 1 ) and at the end (day 13) of training.

The white stars indicate the departure positions. Spatial locations were sampled by means of a grid of resolution $50 \times 50$ (each grid cell is $4 \times 4 \mathrm{~cm}$ ). The value associated to each grid cell was obtained by normalizing the time spent in the cell region with respect to the duration of each trial, and subsequently averaging over all day trials and over all the animals of a group.

On day 1, both groups of mice visited the different alleys of the maze regardless of the starting position (top left and top right). No direct trajectory from the departure point to the platform was observed. After 13 days of training, control mice learned to swim directly from each of the departure points to the platform (bottom left). Similarly, L7-PKCI mice learned to find the location of the platform through direct pathways from each of the four departure points (bottom right).

These plots confirmed that control and mutant mice had a comparable behavior when solving the Starmaze task. 


\section{Supplementary Figure 2}
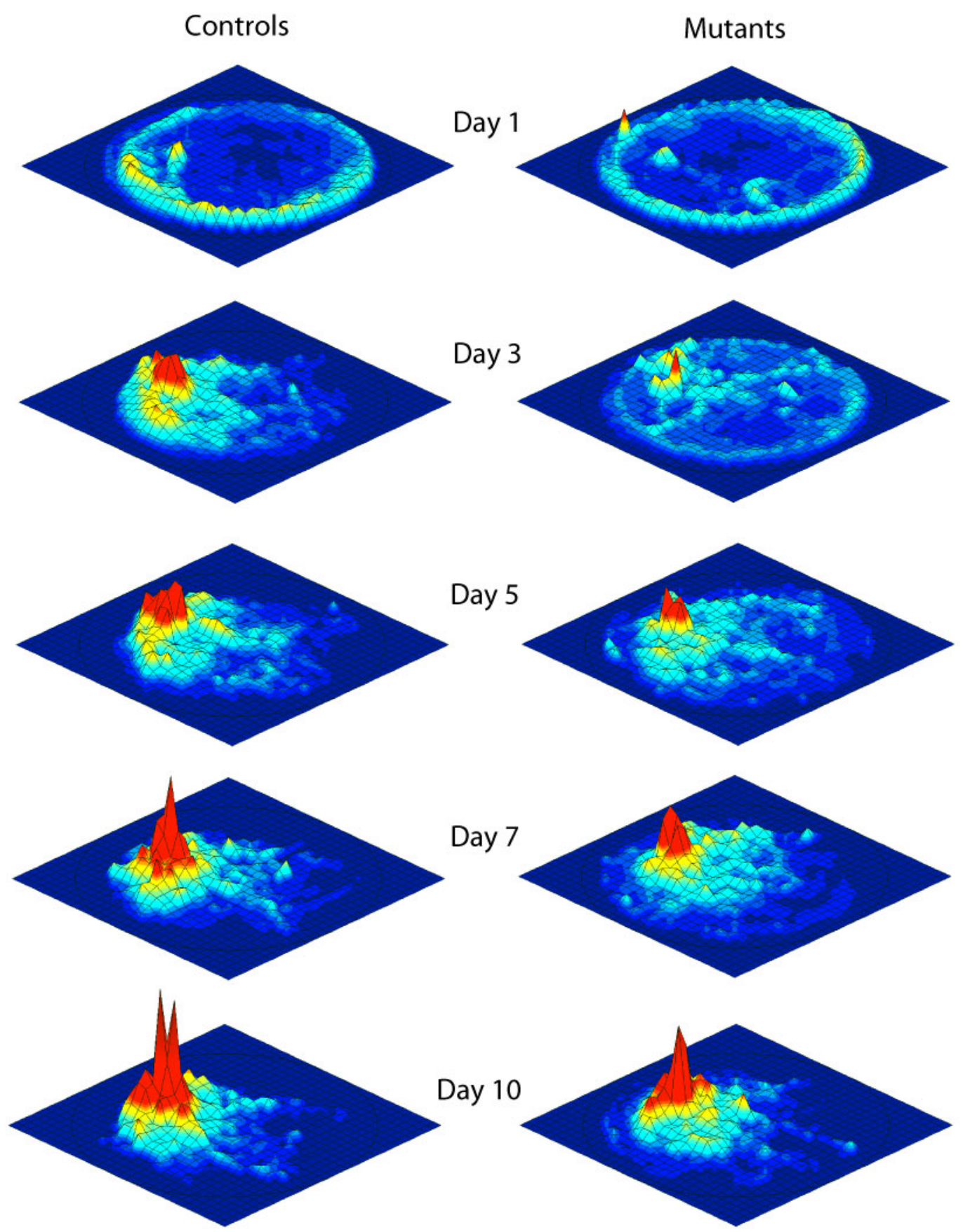

\section{Three-dimensional diagrams of the mean time spent by control and mutant mice at}

each location of the maze at different training phases.

Qualitative representations of the time ( $z$ axis) spent on average by the two groups of animals in the different areas of the pool ( $x$-y axes) at different learning phases (days 1, 3, 5,7 and 10). Spatial locations were sampled by means of a grid of resolution $30 \times 30$ (each grid cell is about $5 \times 5 \mathrm{~cm}$ ). The value associated to each grid cell was obtained by normalizing the time spent in the cell region with respect to the duration of each trial, then averaging over all day trials and over all the animals of a group.

These three-dimensional plots show that, when solving the Morris water maze task, mutant mice had larger searching zones than control animals during the whole learning period. 


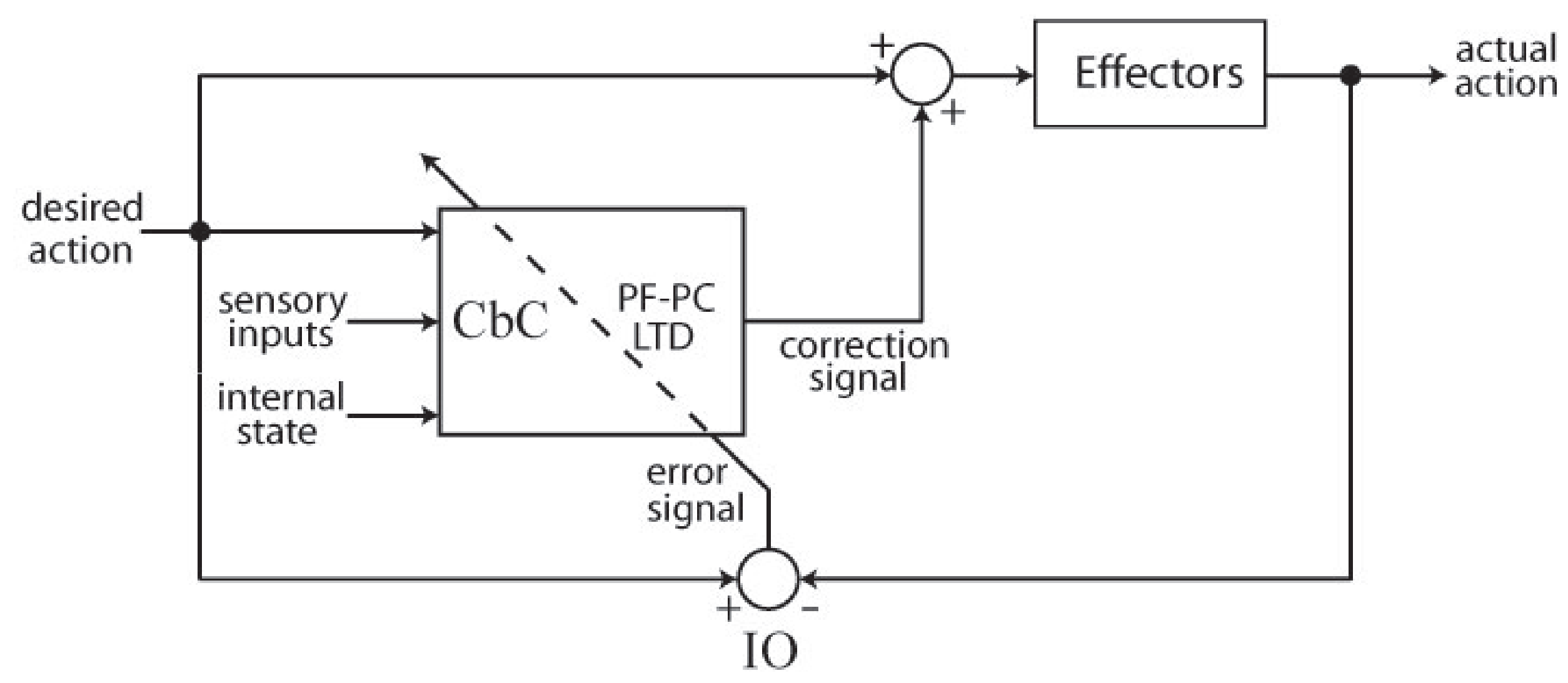

Abstract model describing the error-based cerebellar learning hypothesis (adapted from Ito, M. New concepts in cerebellar function. Rev. Neurol. (Paris) 149, 596-599 (1993)).

This theoretical model can be applied to study the process of sensory-response adaptation underlying both basic motor learning and higher cognitive learning. Contextual information (i.e., multimodal sensory signals, animal's internal state, and desired action) enters the cerebellar cortex $(\mathrm{CbC})$ via the mossy fiber - granule cell - parallel fiber pathway. The inferior olive (IO) generates an error signal based on the discrepancy between desired and actual action, and conveys this information to the cerebellum via the climbing fibers. The convergence of both contextual and error signals at precise timing can induce Long-Term synaptic Depression (LTD) at the parallel fiber - Purkinje cell (PF-PC) synapses. This learning process can lead to the acquisition of the optimal sensory-response linkage and, then, it allows the cerebellum to compensate for the error dynamically. In the case of spatial navigation, this can result in the optimization of the goal-directed trajectory performed by the animal. Specific inactivation of the PF-PC LTD mechanism would impair cerebellar learning and disrupt the correction signal. This would result in non-optimal spatial behavior due to cumulative error over time. 